\title{
Risco de anemia ferropriva em crianças com baixos níveis de ferritina
}

\author{
Risk of iron deficiency anemia in children with low ferritin levels
}

\author{
José Abreu MARTINS-JÚNIOR ${ }^{1}$, Luciana Moreira LIMA² \\ ${ }^{\prime}$ Faculdade de Biomedicina, Universidade José do Rosário Vellano, Belo \\ Horizonte, Brasil. ${ }^{2}$ Departamento de Medicina e Enfermagem, \\ Universidade Federal de Viçosa, Viçosa, Brasil. \\ E-mail:luciana.lima@ufv.br
}

\begin{abstract}
Iron deficiency anemia may impair learning ability in children aged 02 to 06 years. This disease may produce behavioral effects such as lack of attention, fatigue and tiredness that can lead to poor school performance. The aim of this study was to determine the number of children 02 to 06 years who performed the measurements of ferritin, serum iron and hemogram in a clinical laboratory in Belo Horizonte/MG, in the first half 2011, seeking to evaluate the results of these examinations and to establish the prevalence of iron deficiency in studied subjects. We conducted a descriptive cross-sectional observational study. Through software-BI SADIG, used for data management of the clinical laboratory, we selected 363 children from 02 to 06 years who performed the measurements of ferritin, serum iron and hemogram during the period from January first to July thirty-one, two thousand and eleven. The male children had plasma ferritin levels significantly lower when compared with female children $(\mathrm{p}<0.05)$. There were no significant differences between participants of both sexes for serum iron and hemoglobin. Nine children $(2.5 \%)$ had anemia $(\mathrm{Hb}$ $\leq 11.0 \mathrm{~g} / \mathrm{dL}$ and ferritin $\leq 12 \mathrm{ng} / \mathrm{mL}$ ). There was a low prevalence of iron deficiency anemia in children. The most important finding of this study was nearly five times greater risk of developing iron deficiency anemia in children who had plasma ferritin levels below or equal to $12 \mathrm{ng} / \mathrm{mL}$, suggesting that this may be considered the best cutoff point for the population studied.
\end{abstract}

KEY WORDS: iron deficiency anemia; children; ferritin.

\section{RESUMO}

Em crianças pré-escolares a anemia ferropriva pode comprometer a capacidade de aprendizagem, além de produzir efeitos comportamentais como a falta de atenção, fadiga e cansaço que podem levar ao baixo rendimento escolar. $\mathrm{O}$ objetivo deste estudo foi verificar o número de crianças de 02 a 06 anos que realizaram as dosagens de ferritina, ferro sérico e hemograma, com suspeita de anemia ferropriva, num laboratório clínico, no primeiro semestre de 2011, buscando avaliar os resultados dos referidos exames e estabelecer a prevalência de anemia ferropriva nos indivíduos estudados. Foi conduzido um estudo observacional descritivo do tipo transversal. Através do software SADIG-BI, utilizado para gerenciamento de dados, foram selecionadas 363 crianças de 02 a 06 anos que realizaram os exames ferritina, ferro sérico e hemograma no período de 01/01/2011 a 31/07/2011. As crianças do sexo masculino apresentaram níveis plasmáticos de ferritina significativamente mais baixos quando comparadas com as crianças do sexo feminino $(\mathrm{p}<0,05)$. Com relação aos parâmetros ferro sérico e hemoglobina, não foram observadas diferenças significativas entre os participantes dos dois sexos. Das 363 crianças estudadas, 9 (2,5\%) apresentavam anemia ferropriva $(\mathrm{Hb} \leq 11,0$ $\mathrm{g} / \mathrm{dL}$ e Ferritina $\leq 12 \mathrm{ng} / \mathrm{mL}$ ). Foi observada uma baixa prevalência de anemia ferropriva nas crianças estudadas. $\mathrm{O}$ achado mais importante deste estudo foi o risco quase cinco vezes maior do desenvolvimento de anemia ferropriva em crianças que apresentaram níveis plasmáticos de ferritina menores ou iguais a $12 \mathrm{ng} / \mathrm{mL}$, sugerindo que este valor pode ser considerado o melhor ponto de corte para a população estudada

PALAVRAS-CHAVE: anemia ferropriva, crianças, ferritin 


\section{INTRODUÇÃO}

A anemia ferropriva é a deficiência nutricional mais prevalente no homem (1-3). Dados do Ministério da Saúde (MS) apontam que metade dos pré-escolares brasileiros seja anêmica, cerca de 4,8 milhões de crianças, com a prevalência chegando a $67,6 \%$ nas idades entre seis e 24 meses (4). Os dados da Pesquisa Nacional de Demografia e Saúde demonstraram uma prevalência de $20,9 \%$ de crianças menores de 5 anos com hemoglobina $<11 \mathrm{~g} / \mathrm{dL}$, sendo que $8,7 \%$ apresentavam hemoglobina $<9,5 \mathrm{~g} / \mathrm{dL}$ (5).

Estudos realizados com pré-escolares em diversas cidades do Brasil corroboram com dados do MS. Fisberg et al (2001) demonstraram uma prevalência de anemia em $54 \%$ das 2.223 crianças menores de 5 anos estudadas em 10 capitais brasileiras (6). Vieira et al (2001) em estudo realizado com crianças menores de 5 anos de creches públicas da cidade de Recife comprovaram a prevalência de anemia em 55,6\% das crianças estudadas (7). Estudo semelhante realizado com crianças atendidas em creches públicas de São Paulo demonstrou prevalência de anemia em $68,8 \%$ das crianças (8).

Em crianças pré-escolares a doença se torna uma deficiência importante uma vez que compromete a capacidade de aprendizagem, além de produzir efeitos comportamentais como a falta de atenção, fadiga e cansaço que podem levar ao baixo rendimento escolar. Alguns estudos sugerem que a deficiência de ferro em fases precoces da vida pode afetar de maneira irreversível as funções cognitiva e motora (9-11), com diminuição no rendimento motor e nas habilidades cognitivas quando comparadas com as crianças que não apresentaram anemia ferropriva $(12,13)$.

Diante do exposto, torna-se cada vez mais importante a investigação sobre o número de crianças anêmicas bem como sua etiologia com o objetivo de promover intervenções preventivas e de controle. O objetivo deste estudo foi verificar o número de crianças de 02 a 06 anos que realizaram os exames de ferritina, ferro sérico e hemograma, com suspeita de anemia ferropriva, num laboratório clínico em Belo Horizonte/MG, no primeiro semestre de 2011, buscando avaliar os resultados dos referidos exames e estabelecer a prevalência de anemia ferropriva nos indivíduos estudados.

\section{MATERIAIS E MÉTODO}

Por meio do software SADIG-BI, utilizado para gerenciamento de dados do laboratório, foram selecionadas 363 crianças de 02 a 06 anos que realizaram os exames ferritina, ferro sérico e hemograma no período de $01 / 01 / 2011$ a 31/07/2011. Foram consideradas as 28 unidades do laboratório, situadas em diferentes bairros da região metropolitana de Belo Horizonte. O protocolo de estudo recebeu parecer favorável sob o ponto de vista ético e formal pelo Comitê de Ética em Pesquisa da Universidade José do Rosário Vellano (Protocolo no 180/08).

Os exames ferritina, ferro sérico e hemograma foram selecionados por representarem exames muitas vezes solicitados para o diagnóstico de anemia ferropriva, além de representarem os três estágios da deficiência de ferro no organismo humano. Apesar de o hemograma ter sido selecionado, somente o valor da hemoglobina foi considerado. Outros parâmetros, como avaliação dos índices hematimétricos, por exemplo, não foram considerados por não fazerem parte dos objetivos do estudo.

A análise estatística foi realizada utilizando-se os testes t-Student e Mann-Whitney para a comparação de médias. O teste Qui-Quadrado foi utilizado para avaliar a associação entre os fatores de risco (ferro $\leq 35 \mathrm{mcg}$ / $\mathrm{dL}$, ferritina $\leq 45 \mathrm{ng} / \mathrm{mL}$ e ferritina $\leq 12 \mathrm{ng} / \mathrm{mL}$ ) e a presença de anemia $(\mathrm{Hb}<11,0 \mathrm{~g} / \mathrm{dL})$. A associação entre as variáveis contínuas foi analisada pelo teste de correlação de Pearson, considerando-se todos os indivíduos estuda$\operatorname{dos}(\mathrm{n}=363)$. Foi ajustado um modelo de regressão linear múltipla, considerando-se a hemoglobina como variável resposta e todos os demais parâmetros como variáveis independentes. Para todas as análises foi adotado um nível de significância de 5\%. Os programas Sigma Stat versão 1.0 e Prisma versão 3.0 foram utilizados para realizar as análises e confeccionar o gráfico, respectivamente.

\section{RESULTADOS}

A Tabela 1 apresenta a análise descritiva e resultados obtidos quando os participantes foram divididos por sexo.

Participaram do estudo 363 crianças que realizaram os exames ferritina, ferro sérico e hemograma no período de Janeiro a Junho de 2011. Dessas, 202 (55,6\%) eram do sexo masculino e $161(44,4 \%)$ do sexo feminino. As crianças do sexo masculino apresentaram níveis plasmáticos de ferritina significativamente mais baixos quando comparadas com as crianças do sexo feminino $(p<0,05)$. Com relação aos parâmetros ferro sérico e hemoglobina, não foram observadas diferenças significativas entre os participantes dos dois sexos.

A Figura 1 apresenta a distribuição dos valores observados para os níveis plasmáticos de ferritina de acordo com o sexo das crianças estudadas. Foram observadas correlações positivas e significativas entre os parâmetros ferro e hemoglobina $(r=0,24 ; \mathrm{p}<0,001)$, ferro e ferritina $(\mathrm{r}=0,12 ; \mathrm{p}<0,001)$, ferro e idade $(\mathrm{r}=0,19$; $\mathrm{p}<0,001)$, hemoglobina e idade $(\mathrm{r}=0,14 ; \mathrm{p}=0,01)$ e entre ferritina e idade $(\mathrm{r}=0,12 ; \mathrm{p}=0,03)$, considerando-se os 363 participantes. 
Tabela 1 - Análise descritiva e resultados de hemograma de crianças participantes do estudo.

\begin{tabular}{|c|c|c|c|c|}
\hline & Total & Sexo masculino & Sexo feminino & $\mathbf{p}$ \\
\hline$n$ & $363(100 \%)$ & $202(55,6 \%)$ & $161(44,4 \%)$ & - \\
\hline Idade (anos) & $3,6 \pm 1,4$ & $3,5 \pm 1,8$ & $3,0 \pm 1,7$ & 0,431 \\
\hline $\begin{array}{l}\text { Hemoglobina }(\mathrm{g} / \mathrm{dL}) \\
\text { Média } \pm \text { Desvio padrão }\end{array}$ & $12,5 \pm 0,9$ & $12,4 \pm 9,8$ & $12,5 \pm 9,9$ & 0,335 \\
\hline $\begin{array}{l}\text { Hemoglobina }(\mathrm{g} / \mathrm{dL}) \\
\text { Mediana (intervalo interquartil) }\end{array}$ & $12,5(11,9-13,1)$ & $12,4(11,9-13,1)$ & $12,6(11,9-13,1)$ & 0,333 \\
\hline $\begin{array}{l}\text { Ferro }(\mathrm{mcg} / \mathrm{dL}) \\
\text { Média } \pm \text { Desvio padrão }\end{array}$ & $72,6 \pm 33,4$ & $71,6 \pm 33,1$ & $73,9 \pm 33,9$ & 0,509 \\
\hline $\begin{array}{l}\text { Ferro (mcg/dL) } \\
\text { Mediana (intervalo interquartil) }\end{array}$ & $68,0(49,0-93,0)$ & $68,0(49,0-90,0)$ & $67,0(48,8-97,2)$ & 0,601 \\
\hline $\begin{array}{l}\text { Ferritina }(\mathrm{ng} / \mathrm{mL}) \\
\text { Média } \pm \text { Desvio padrão }\end{array}$ & $27,0 \pm 22,9$ & $23,6 \pm 19,1$ & $31,2 \pm 26,5$ & $0,002^{(*)}$ \\
\hline $\begin{array}{l}\text { Ferritina ( } \mathrm{ng} / \mathrm{mL} \text { ) } \\
\text { Mediana (intervalo interquartil) }\end{array}$ & $21,1(13,9-33,5)$ & $19,8(13,2-28,2)$ & $23,8(15,6-37,9)$ & $<0,0001^{(\dagger)}$ \\
\hline
\end{tabular}

Caracterização do grupo e análise comparativa de acordo com o sexo. $\mathrm{n}=$ tamanho da amostra; $\mathrm{p}$ - probabilidade para os testes de hipóteses; $\left({ }^{*}\right)$ teste t-Student; $\left({ }^{\dagger}\right)$ teste Mann-Whitney.

Entre os demais parâmetros não foram observadas correlações.

Os dados obtidos com a análise da associação entre os fatores de risco para anemia ferropriva e níveis de hemoglobina $(\mathrm{Hb}) \leq 11,0 \mathrm{~g} / \mathrm{dL}$ são apresentados na Tabela 2. Das 363 crianças estudadas, $9(2,5 \%)$ apresentavam anemia ferropriva $(\mathrm{Hb} \leq 11,0 \mathrm{~g} / \mathrm{dL}$ e Ferritina $\leq 12 \mathrm{ng} / \mathrm{mL})$. A Tabela 2 também apresenta os valores de odds ratios obtidas para os grupos $\mathrm{Hb} \leq 11,0 \mathrm{~g} / \mathrm{dL}$ e $\mathrm{Hb}>11,0 \mathrm{mg} / \mathrm{dL}$ de acordo com os fatores de risco para anemia.

Figura 1 - Distribuição dos valores observados para os níveis plasmáticos de ferritina em crianças de acordo com o sexo

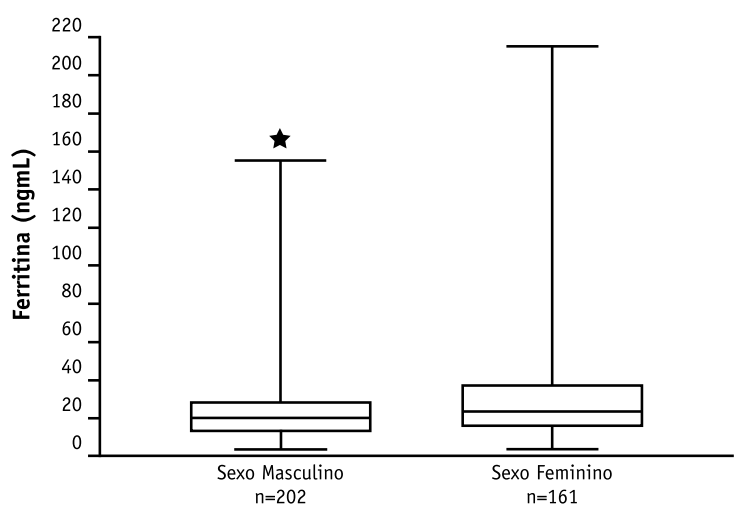

Valores expressos em $\mathrm{ng} / \mathrm{mL}$ para participantes do sexo masculino e feminino. Cada box representa a mediana, primeiro e terceiro quartis dos grupos, $(\star)$ indica diferença significativa em relação aos participantes do sexo feminino $(\mathrm{p}<0,0001)$.
Os participantes que apresentaram níveis de ferritina $\leq 12,0 \mathrm{ng} / \mathrm{mL}$ apresentaram um risco significativamente maior de anemia (hemoglobina $\leq 11,0 \mathrm{~g} / \mathrm{dL}$ ) do que os participantes com níveis de ferritina maiores que $12,0 \mathrm{ng} / \mathrm{dL}$ (odds ratio 4,6; IC 1,93-10,91; $\mathrm{p}=0,002$ ).

\section{DISCUSSÃO}

A ferritina sérica é um parâmetro utilizado para avaliar o estoque de ferro corporal, sendo considerada medida útil por utilizar sangue periférico e apresentar forte correlação com o ferro em depósito nos tecidos $(14,15)$. Segundo Neves et al $(2005)$ a ferritina é um bom indicador da deficiência de ferro por possuir alta especificidade na caracterização da anemia ferropriva (16). As únicas situações clínicas associadas à diminuição de ferritina sérica com depósitos normais de ferro são o hipotireoidismo e a deficiência de vitamina C (17). Por outro lado, na presença de infecções, neoplasias, doenças hepáticas, leucemias, ingestão de álcool e hipertireoidismo esses valores podem se apresentar elevados (18). Hadler et al (2002), utilizando a ferritina como parâmetro, demonstraram entre 35 lactentes não anêmicos, a presença de $20 \%$ com depleção das reservas de ferro (19). Não existe consenso quanto ao ponto de corte para caracterizar o indivíduo com depleção das reservas de ferro. Alguns estudos têm adotado valores inferiores a $12 \mathrm{mg} / \mathrm{L}(6,15,18)$. Killip et al (2007) utilizaram valores de ferritina inferiores a $45 \mathrm{mg} / \mathrm{L}$ para a caracterização da depleção das reservas de ferro (14) sendo que cada $\mathrm{mg} / \mathrm{L}$ de ferritina sérica representa cerca de 8 a $10 \mathrm{mg}$ de ferro armazenado (20). 
$\mathrm{Na}$ análise dos dados deste estudo foi feita a opção der utilizar valores de ferritina inferiores a $12 \mathrm{mg} / \mathrm{L}$ na caracterização da depleção das reservas de ferro, uma vez que os dados da Tabela 2 mostraram que os partici- pantes com níveis de ferritina menores que $12,0 \mathrm{ng} / \mathrm{mL}$ apresentaram um risco 4,6 vezes maior de ocorrência de anemia (hemoglobina $\leq 11,0 \mathrm{~g} / \mathrm{dL}$ ) do que os participantes com níveis de ferritina maiores que $12,0 \mathrm{ng} / \mathrm{dL}$.

Tabela 2 - Odds ratios obtidas para os grupos $\mathrm{A}(\mathrm{Hb} \leq 11,0 \mathrm{~g} / \mathrm{dL})$ e $\mathrm{B}(\mathrm{Hb}>11,0 \mathrm{mg} / \mathrm{dL})$ de acordo com os fatores de risco para anemia ferropriva

\begin{tabular}{|c|c|c|c|c|c|}
\hline Grupos & $\mathbf{n}$ & $\begin{array}{c}\text { Ferro } \leq 35 \mathrm{mcg} / \mathrm{dL} \\
\mathrm{n}(\%)\end{array}$ & $\begin{array}{c}\text { Ferro }>35 \mathrm{mcg} / \mathrm{dL} \\
\mathrm{n}(\%)\end{array}$ & Odds Ratio ${ }^{1}$ & $p$ \\
\hline A & 25 & $3(12)$ & $22(88)$ & --- & --- \\
\hline \multirow[t]{2}{*}{ B } & 338 & $34(10)$ & $304(90)$ & $1,219(0,371-4,040)$ & 0,757 \\
\hline & & Ferritina $\leq 45 \mathrm{ng} / \mathrm{mL}$ & Ferritina $>45 \mathrm{ng} / \mathrm{mL}$ & & \\
\hline A & 25 & $21(84)$ & $4(16)$ & --- & --- \\
\hline \multirow[t]{2}{*}{ B } & 338 & $300(89)$ & $38(11)$ & $0,665(0,226-1,944)$ & 0,473 \\
\hline & & Ferritina $\leq 12 \mathrm{ng} / \mathrm{mL}$ & Ferritina $>12 \mathrm{ng} / \mathrm{mL}$ & & \\
\hline A & 25 & $9(36)$ & $16(64)$ & --- & --- \\
\hline B & 338 & $37(11)$ & $301(89)$ & $4,576(1,927-10,906)$ & 0,002 \\
\hline
\end{tabular}

$\mathrm{Hb}$ : hemoglobina; ${ }^{1}$ Intervalo de confiança $95 \%$

O mesmo não aconteceu quando foi considerado o ponto de corte para os níveis de ferritina em 45,0 ng/ dL, não sendo observada significância estatística. Esses dados indicam que o melhor ponto de corte para os níveis plasmáticos de ferritina foi de $12,0 \mathrm{ng} / \mathrm{dL}$ para os indivíduos estudados.

Este estudo não demonstrou associação entre níveis de ferro sérico menores que $35 \mathrm{mcg} / \mathrm{dL}$ e risco aumentado de hemoglobina $\leq 11,0 \mathrm{~g} / \mathrm{dL}$ (Tabela 2 ). A dosagem de ferro normalmente é utilizada para medir a quantidade de ferro ligado à transferrina (proteína de transporte) em plasma sanguíneo. Quando as reservas de ferro estão baixas, qualquer declínio adicional no ferro corporal é acompanhado por uma redução na concentração do ferro sérico. Devido às alterações diárias do nível de ferro sérico (21), este teste isoladamente apresenta valor limitado, devendo ser analisado em combinação com a saturação da transferrina e a ferritina sérica (22).

Os dados ora apresentados mostram que, apesar do número de crianças anêmicas $(\mathrm{Hb} \leq 11,0 \mathrm{~g} / \mathrm{dL}$ e Ferritina $\leq 12 \mathrm{ng} / \mathrm{mL})$ parecer pequeno, $9(2,5 \%)$ crianças das 363 estudadas, ele se torna relevante se considerado que nesse estudo foram analisadas apenas crianças que realizaram os três exames (ferritina, ferro sérico e hemograma) em uma instituição particular. Outros estudos mostraram que a anemia ferropriva, apesar de não ser uma doença exclusiva dos países em desenvolvimento, apresenta uma estreita relação com a condição sócio-econômica da população $(23,24)$.

Apesar dos coeficientes de correlação demonstrados por este estudo serem baixos, a correlação obtida entre a idade e níveis de ferro (19\%), ferritina $(12 \%)$ e hemoglobina (14\%) podem corroborar com os dados do estudo de Vieira et al (2007), que demonstraram correlação positiva de hemoglobina e ferritina com a idade das crianças avaliadas (7).

Os dados obtidos com o presente estudo mostraram uma prevalência de $2,5 \%$ de anemia ferropriva nas crianças estudadas, considerando os níveis de hemoglobina menores que $11,0 \mathrm{~g} / \mathrm{dL}$ e os níveis de ferritina menores que $12 \mathrm{ng} / \mathrm{dL}$. Se considerados apenas os níveis de hemoglobina menores que $11 \mathrm{~g} / \mathrm{dL}$, a prevalência de anemia foi de $6,9 \%$. Os estudos conduzidos com a população brasileira apontam elevada prevalência de anemia ferropriva em crianças dependendo da região e da faixa etária estudada (1). Neves et al (2005), em estudo de prevalência de anemia em lactentes utilizando os parâmetros hemoglobina e ferro sérico observaram presença de $55,1 \%$ de anêmicos nos 365 lactentes estudados (16). Apesar de existirem inúmeros trabalhos publicados sobre a prevalência da anemia ferropriva no Brasil e no mundo e já se ter concluído que a anemia ferropriva constitui um grave problema de saúde pública (25), ainda assim o número de crianças com essa deficiência é enorme. 


\section{CONCLUSÕES}

Foi observada uma baixa prevalência de anemia ferropriva nas crianças estudadas. O achado mais importante deste estudo foi o risco quase cinco vezes maior do desenvolvimento de anemia ferropriva em crianças que apresentaram níveis plasmáticos de ferri- tina menores ou iguais a $12 \mathrm{ng} / \mathrm{mL}$, sugerindo que este valor pode ser considerado o melhor ponto de corte para a população estudada. Estudos adicionais, envolvendo um maior número de crianças podem contribuir para esclarecer, com fidedignidade, o ponto de corte a ser adotado para os níveis plasmáticos de ferritina em crianças de dois a seis anos.

\section{REFERÊNCIAS}

1. Braga JAP, Vitalle MSS. Deficiência de ferro na criança. Rev Bras Hematol Hemoter. 2010;32(supl 2):38-44.

2. Vicari P, Figueiredo MS. Diagnóstico diferencial da deficiência de ferro. Rev Bras Hematol Hemoter. 2010;32(supl 2):29-31.

3. Polin V, Coriat R, Perkins G, Dhooge M, Abitbol V, Leblanc S, Prat F, Chaussade S. Iron deficiency: From diagnosis to treatment. Dig Liver Dis. 2013 Apr 10. [Epub ahead of print].

4. BRASIL. Ministério da Saúde. Portaria $n^{\circ} 730$, de 13 de maio de 2005. Institui o Programa Nacional de Suplementação de Ferro, destinado a prevenir a anemia ferropriva e dá outras providências. 2005.

5. BRASIL. Ministério da Saúde. Centro Brasileiro de Análise e Planejamento. Pesquisa Nacional de Demografia e Saúde da Criança e da Mulher PNDS, 2006. Série G. Estatística e Informação em Saúde. 2009.

6. Fisberg M, Naufel C, Braga JAP. National prevalence of anaemia in preschool children in Brazil: 10 capitais survey [abstract]. Ann Nutr Metab. 2001;45(suppl 1):450

7. Vieira ACF, Diniz AS, Cabral PC, Oliveira RS, Lola MMF, Silva SMM et al. Avaliação do estado nutricional de ferro e anemia em crianças menores de 5 anos de creches públicas. J. Pediatria. 2007;83(4):370-6

8. Bueno MB, Selem SSC, Arêas JAG, Fisberg MR Prevalência e fatores associados à anemia entre crianças atendidas em creches públicas de São Paulo. Rev Bras Epidem. 2006;9(4):462-70.

9. Brunken GS, Guimarães LV, Fisberg M. Anemia em crianças menores de 3 anos que freqüentam creches públicas em período integral. J Pediatria. 2002;78(1):50-6.

10. Camillo CC, Amancio OMS, Vitalle OMS, Braga JAP, Juliano Y. Anemia ferropriva e estado nutricional de crianças de creches de Guaxupé. Rev Assoc Med Bras. 2008;54(2):154-9.

11. Compri PC, Cury MCFS, Novo NF, Juliano Y, Sigulem DM.Variáveis maternas e infantis associadas à ocorrência de anemia em crianças nos serviços de atenção básica em São Paulo. Rev Paulista Pediatria. 2007;25(4):349-54.

12. Heijblom GS, Santos LMP. Anemia ferropriva em escolares da primeira série do ensino fundamental da rede pública de educação de uma região de Brasília, DF. Rev Bras Epidem. 2007;10(2):258-66.
13. Santos CD, Santos LMP, Figueiroa JN, Marroquim PMG, Oliveira MAA. Anemia em escolares da $1^{\mathrm{a}}$ série do ensino fundamental da rede pública de Maceió - AL. Cad Saúde Pública. 2002;18(6):1757-63.

14. Killip S, Bennett JM, Chambers MD. Iron Deficiency Anemia. Am Family Physician. 2007;75(5):671-8.

15. Grotto HZW. Fisiologia e metabolismo do ferro. Rev Bras Hematol Hemoter. 2010;32(supl 2):8-17.

16. Neves MBP, Silva EMK, Morais MB. Prevalência e fatores associados à deficiência de ferro em lactentes atendidos em um centro de saúde-escola em Belém, Pará, Brasil. Cad. Saúde Pública. 2005;21(6):1911-8.

17. Zago MA, Falcão RP, Pasquin R. Hematologia: fundamentos e prática. 1 ed. São Paulo: Atheneu, 2001.

18. Paiva AA, Rondó PHC, Guerra-Shinohara EM. Parâmetros para avaliação do estado nutricional de ferro. Rev. Saúde Pública. 2000;34(4):421-6.

19. Hadler MC, Juliano Y, Sigulem DM. Anemia do lactente: etiologia e prevalência. J. Pediatria. 2002;78(4):321-6.

20. Cook JD. Clinical evaluation of iron deficiency. Semin Hematol. 1982;19(1):6-18.

21. Dale JC, Burritt MF, Zinsmeister AR. Diurnal variation of serum iron, iron-binding capacity, transferrin saturation, and ferritin levels. Am J Clin Pathol. 2002;117(5):802-8.

22. Grotto HZW. Diagnóstico laboratorial da deficiência de ferro. Rev Bras Hematol Hemoter. 2010;32(supl 2):22-8.

23. Santos I, César JA, Minten G, Valle N, Neumann NA, Cercato E. Prevalência e fatores associados à ocorrência de anemia entre menores de seis anos de idade em Pelotas, RS. Rev Bras Epidemiol. 2004;7(4):403-15.

24. Silva LSM, Giuliani ERJ, Aerts DRGC. Prevalência e determinantes de anemia em crianças de Porto Alegre, RS, Brasil. Rev Saúde Pública. 2001;35(1):66-73.

25. Szarfarc SC. Políticas públicas para o controle da anemia ferropriva. Rev Bras Hematol Hemoter. 2010;32(supl 2):2-8. 